\title{
Full Mouth Rehabilitation in Dental Fluorosis Patient - A Time Restrained Scenario
}

\author{
Saloni Kachhara ${ }^{1}$, Ashok V. ${ }^{2}$ \\ ${ }^{1}$ Department of Prosthodontics and Implant Dentistry, Saveetha Dental College, SIMATS, Chennai, Tamilnadu, India. \\ ${ }^{2}$ Department of Prosthodontics and Implant Dentistry, Saveetha Dental College, SIMATS, Chennai, Tamilnadu, India.
}

\section{INTRODUCTION}

Dental Fluorosis which is endemic in nature is considered to be a serious problem in nearly 15 states of India. ${ }^{1}$ It is defined as hypo-mineralization of enamel resulting from excessive ingestion of fluoride (more than $1 \mathrm{ppm}$ ) during tooth development. Excessive fluoride basically disturbs mineralization in the developing tooth by decreasing free calcium ion concentrations in the mineralizing matrix. In turn, it indirectly interferes with the proteinases which degrade matrix proteins during the maturation phase of amelogenesis, ${ }^{2}$ resulting in delayed enzymatic degradation of enamel matrix proteins. ${ }^{3}$ The fluoride-induced retention of enamel matrix proteins is responsible for impaired and incomplete crystal growth resulting in the increased porosity of enamel, and the subsequent optical and physical changes. ${ }^{4}$

The severity of fluorosis depends on the duration, frequency and timings of the exposure to fluoride during tooth development. ${ }^{5}$ In its mildest form, enamel fluorosis appears as loss of marginal translucency, faint white flecks, spots or striations. With increasing severity, white flecks or striations enlarge and may merge, ${ }^{4}$ while the classical appearance of fluorosis is characterized by banding following the developmental lines of enamel and by substantial symmetry on homologous teeth. ${ }^{6}$ In its severest form, hypo-mineralization extends towards the dentinal-enamel junction, and may result in dark brown to black stains. 7,8

Thylstrup and Fejerskov 9 gave their classification of fluorotic teeth with 9 different grades in the increasing order of severity. Other indices described to classify dental fluorosis in the literature are the tooth surface fluorosis index.9,10 fluorosis risk index ${ }^{11}$ and the Dean's Fluorosis Index ${ }^{12}$ which is most widely used.

The treatment options for managing dental fluorosis range from simple restorative procedures like bleaching and composite fillings to complex procedures like veneers and crowns including the full mouth rehabilitation. Full mouth rehabilitation is considered to be one of the biggest challenges to any clinician. It needs efficient diagnosis and thorough treatment planning to develop correct occlusion to optimize stomatognathic function, health and aesthetics which in turn leads to patient's comfort and satisfaction. ${ }^{13}$

This article documents a case of severe dental fluorosis in a 48-year-old male patient with chief complaint of bad aesthetics and also a very crucial time constraint of 20 days. Full mouth rehabilitation was planned and performed for him in 20 days. The aim of the case report was to bring forts the new clinical and technical alterations to simplify the procedure of full mouth rehabilitation in a time restrained scenario.

The patient was extremely happy and satisfied with the new smile. It boosted his confidence and improved his social life.

\author{
Corresponding Author: \\ Saloni Kachhara, \\ Postgraduate Student, \\ Department of Prosthodontics \& \\ Implant Dentistry, Saveetha Dental College, \\ Saveetha Institute of Medical and Technical \\ Sciences, 162, Poonamallee High Road, \\ Chennai-600077, Tamilnadu, India. \\ E-mail:drsbk25@gmail.com
}

DOI: $10.14260 /$ jemds/2020/321

Financial or Other Competing Interests: None.

How to Cite This Article:

Kachhara S, Ashok V. Full mouth rehabilitation in dental fluorosis patient- a time restrained scenario. J. Evolution Med. Dent. Sci. 2020;9(17):1471-1474, DOI: $10.14260 /$ jemds $/ 2020 / 321$

Submission 03-02-2020,

Peer Review 11-04-2020,

Acceptance 18-04-2020,

Published 27-04-2020.

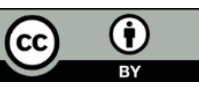




\section{PRESENTATION OF CASE}

A 48-year-old gentleman reported to the department of Prosthodontics and Implantology with a complaint of severe discoloration of front teeth. According to the patient, the discolouration was prominent since childhood in both primary and permanent dentition. A detailed personal history revealed that the patient belonged to one of the fluoride belts of India and had only well water during his childhood. His medical history was non-contributory and the patient was neither allergic to medicine or food, nor addicted to drugs or smoking. Patient was permanently shifting to Dubai in next 20 days and wanted the treatment in that time span.

On clinical examination, there was generalized brown hue in all the teeth with dark brown bands covering the cervical to middle third of all the teeth along with enamel pitting (Fig. 1). There were amalgam restorations in 35,45 and 14, glass ionomer restoration in 17, caries in 36, 26 and 27. 16, 24, 25, 37 and 47 teeth were missing. The occlusion was fine and there was no loss in vertical dimension. Mild attrition was present on 36, 46 and 26. A diagnosis of 'generalized severe fluorosis' was made based on the patient's history and clinical presentation.

\section{DISCUSSION OF MANAGEMENT}

Addressing the chief complaint of the patient which was discoloration, metal ceramic full coverage crowns were decided for the patient. Metal-ceramic crown was chosen as it would completely cover the dark brown shade of the teeth. The missing teeth could be replaced by two options: fixed partial denture or implant supported crowns. Due to time crunch and also since all the teeth were to be prepared for giving crowns, fixed partial dentures were planned for the patient in maxillary posterior region.

The entire treatment was completed in 7 appointments as follows-

- $\quad$ Appt. 1: Photographs (with patient's consent), Diagnostic impressions with irreversible hydrocolloid (Zhermack, Tropicalgin), Facebow transfer for tentative mounting.

- Appt. 2: Anterior tooth preparations and temporization.

- Appt. 3: Posterior tooth preparations, Master impressions of maxillary and mandibular arches with vinyl polysiloxane material (Zhermack elite $\mathrm{HD}+$ ) and temporization. (fig. 2,3)

- $\quad$ Appt. 4: Face-bow record and jaw relation (Fig. 4)

- Appt. 5: Metal trial. (fig. 5)

- Appt. 6: Ceramic bisque trial.

- Appt. 7: Final cementation and maxillary arch impression. (fig. 6)

The diagnostic impressions were poured in dental stone (Kalabhai, Kalstone) and two sets of casts were made. One set of casts was mounted in semi-adjustable articulator (Stratos 300) and wax up was done only for the missing teeth. Mock preparations of all teeth were done on the second set of casts and temporaries were fabricated by indirect method. The master impressions were made by double stage putty light body impression technique with double cord packing (Ultradent, Ultrapak Cord \#00 and \#0). The final jaw relation was recorded using the temporaries and pattern resin technique. The master impressions were poured in die stone (Kalabhai, Ultrarock) and were mounted on the semiadjustable articulator (Stratos 300). Full mouth RVGs were taken on the appointment of metal trial to check for the fit of the metal. Since all the canines were present, canine-guided occlusion was planned for the patient. The final prosthesis was cemented using glass ionomer cement for luting (Shofu Inc, HY bond GIC). In the final step, a hard-acrylic stabilization splint (Michigin splint) for the maxillary arch was provided to the patient for wearing at night for a couple of weeks.
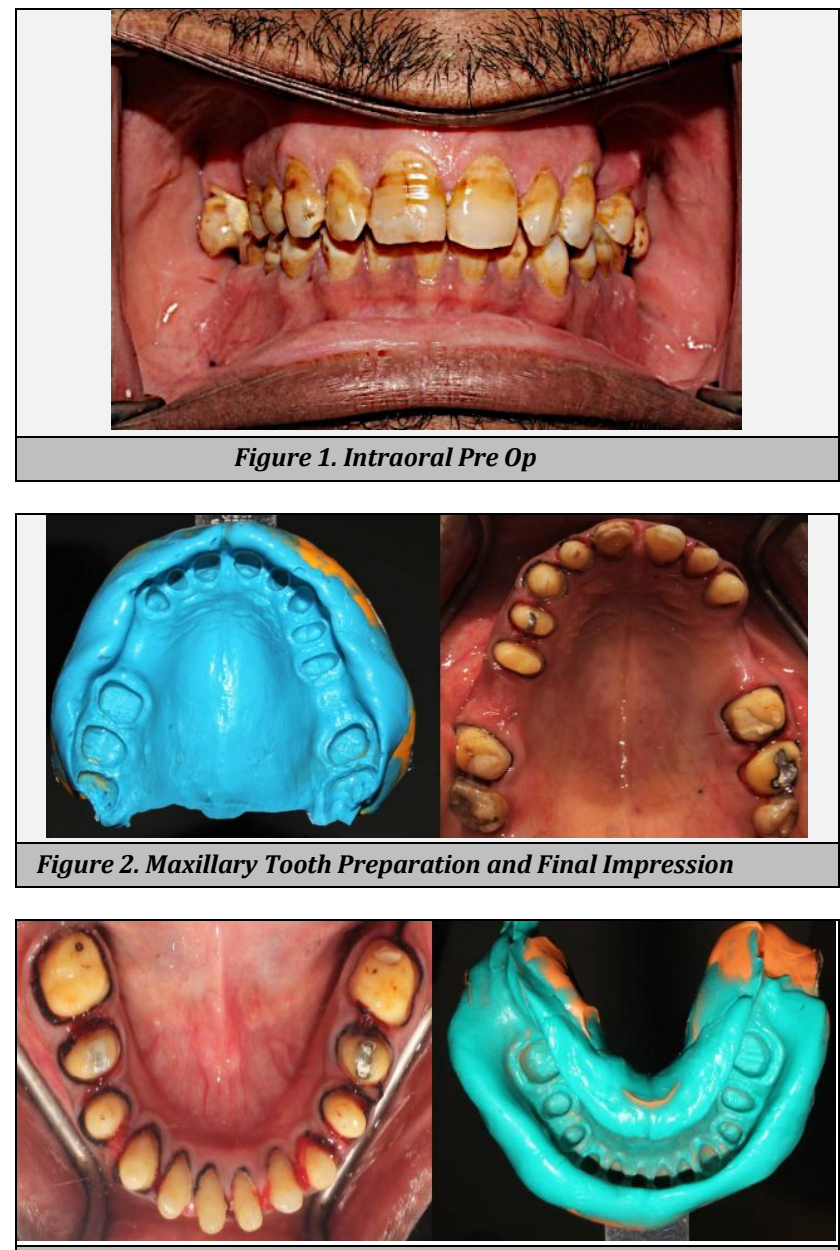

Figure 3. Mandibular Tooth Preparation and Final Impression

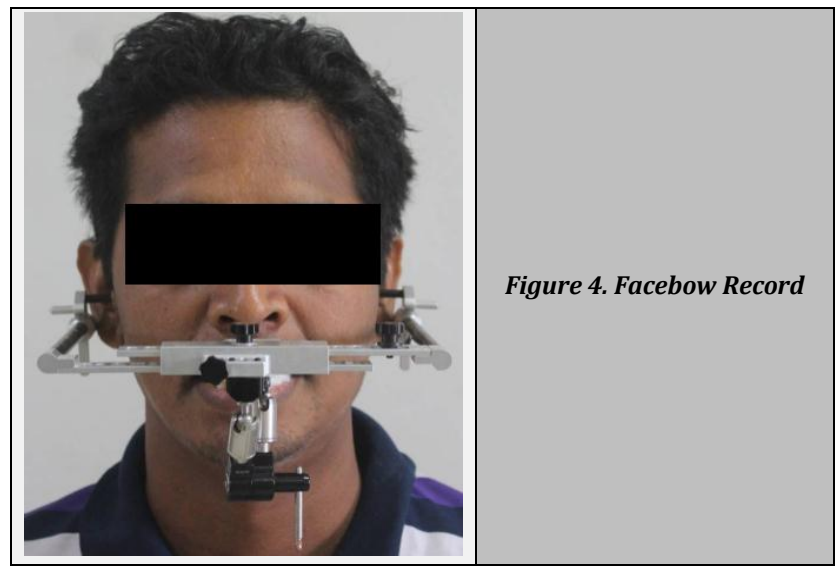



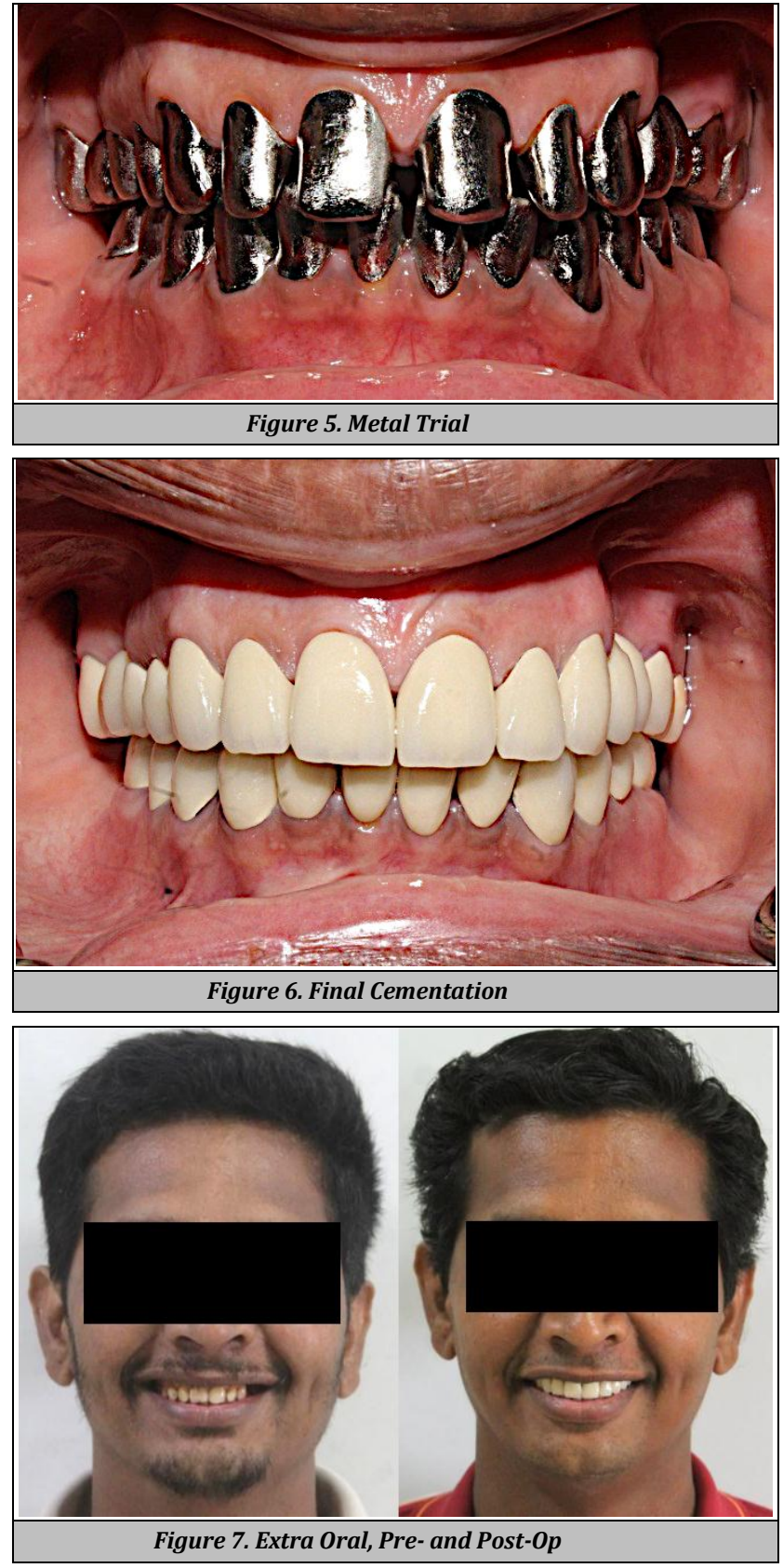

DISCUSSION

Pleasant smile aesthetics is an important contributory factor to the psychosocial well-being of a person. A pleasant smile makes the person confident and boosts his performance. ${ }^{14}$ Teeth discoloration due to fluorosis is an aesthetic concern for most of the patients. Treatment options depend upon the severity of condition as well as patient's aesthetic demands. 5,15 A variety of restorative treatment options are available. Bleaching alone or in combination with micro/macro abrasion is the most conservative approach for mild-and-moderate fluorosis. ${ }^{15}$ However they cannot be considered as an ultimate solution for severe fluorosis cases. In these conditions, either veneers or full coverage crowns are needed to satisfy the patient's aesthetic demands. ${ }^{12}$

Fixed Prosthodontic procedures are of two types: Conformative or Re-organized. In conformative approach, the centric of the patient is not changed and the same occlusion is followed after giving the prosthesis. In the re-organized approach, a new centric is obtained for the patient using a deprogrammer and the prosthesis is fabricated on the new record established. ${ }^{16}$ Since there was no loss in the vertical dimension and the patient had stable centric relation a conformative approach was followed for the case.

The dentition in full occlusal rehabilitation cases are restored following different principles and philosophies of the occlusal schemes. The two basic schemes followed are canine guided occlusion or the group function. ${ }^{17}$ The canine guided occlusion is the favoured occlusal scheme in most of the full mouth rehabilitation cases. ${ }^{18,19}$ Since both the natural canines were present in the maxillary as well as mandibular arch, a canine guided occlusal scheme was finalized for the patient in whom the posteriors occlude only in the centric relation and are in dis-occlusion in any eccentric movements. During protrusive movement, only the anteriors contact while during laterotrusive movements there is contact only on the canines.

The main aim of any clinician should be to satisfactorily meet the demands of his patient while maintaining the basic requirements of health care. ${ }^{20}$ In the present case, the patient was not confident of his smile and this in turn it affected his psycho-social well- being. The full mouth rehabilitation treatment boosted patient's confidence and also made him look younger than his age. (fig. 7) The present case is an example of stepwise full-mouth rehabilitation utilizing different restorative approaches to get an aesthetically, as well as functionally, acceptable outcome. The technique of full occlusal rehabilitation illustrated here simplifies the procedures while adhering to all its principles in the stipulated period of time.

\section{REFERENCES}

[1] Teotia SP, Teotia M. Endemic fluorosis in India: a challenging national health problem. J Assoc Physicians India 1984;32(4):347-52.

[2] Aoba T, Fejerskov 0. Dental fluorosis: chemistry and biology. Critical Reviews in Oral Biology \& Medicine 2002;13(2):155-70.

[3] Ng F, Manton DJ. Aesthetic management of severely fluorosed incisors in an adolescent female. Australian Dental Journal 2007;52(3):243-8.

[4] Robinson C, Connell S, Kirkham J, et al. The effect of fluoride on the developing tooth. Caries Research 2004;38(3):268-76.

[5] Farid H, Khan FR. Clinical management of severe fluorosis in an adult. BMJ Case Reports 2012;2012:bcr2012007138.

[6] Levy SM. An update on fluorides and fluorosis. J Can Dent Assoc 2003;69(5):286-91.

[7] DenBesten PK. Biological mechanisms of dental fluorosis relevant to the use of fluoride supplements. Community Dentistry and Oral Epidemiology 1999;27(1):41-7.

[8] Pandit N, Jadav P. Fluorosis - an update and review. Journal of Integrated Health Sciences 2013;1(1):65-7.

[9] Thylstrup A, Fejerskov 0. Clinical appearance of dental fluorosis in permanent teeth in relation to histologic changes. Community Dentistry and Oral Epidemiology 1978;6(6):315-28. 
[10] Horowitz HS, Driscoll WS, Meyers RJ, et al. A new method for assessing the prevalence of dental fluorosis-the tooth surface index of fluorosis. The Journal of the American Dental Association 1984;109(1):37-41.

[11] Pendrys DG. The fluorosis risk index: a method for investigating risk factors. Journal of Public Health Dentistry 1990;50(5):291-8.

[12] Sherwood IA. Fluorosis varied treatment options. J Conserv Dent 2010;13(1):47-53.

[13] Sudhir N, Prakash H. Full mouth rehabilitation with group function occlusal scheme in a patient with severe dental fluorosis. Indian Journal of Dental Advancements 2011;3(3):627-31.

[14] Lukez A, Pavlic A, Zrinski TM, et al. The unique contribution of elements of smile aesthetics to psychosocial well-being. J Oral Rehabil 2015;42(4):27581.
[15] Pandey P, Ansari AA, Moda P, et al. Enamel microabrasion for aesthetic management of dental fluorosis. BMJ Case Reports 2013;2013:bcr2013010517.

[16] Davies S. Conformative, re-organized or unorganized? Dental Update 2004;31(6):334-45.

[17] McAdam DB. Tooth loading and cuspal guidance in canine and group-function occlusions. J Prosthet Dent 1976;35(3):283-90.

[18] Stuart CE. Good occlusion for natural teeth. The Journal of Prosthetic Dentistry 1964;14(4):716-24.

[19] Stuart CE, Stallard H. Principles involved in restoring occlusion to natural teeth. The Journal of Prosthetic Dentistry 1960;10(2):304-13.

[20] Sabin JE. Clinical skills for the 1990s: six lessons from HMO practice. Hosp Community Psychiatry 1991;42(6):605-8. 